\title{
Waveform moment analysis: An ASYST program for topographical analyses of nonnegative bounded waveforms
}

\author{
JOHN T. CACIOPPO \\ Ohio State University, Columbus, Ohio \\ and \\ BEVERLY S. MARSHALL-GOODELL, LOUIS G. TASSINARY, FUCHIH CHEN, \\ STACY J. SIEGEL, and DONALD D. DORFMAN \\ University of Iowa, Iowa City, Iowa
}

\begin{abstract}
The moments of a nonnegative bounded waveform (e.g., bounded probability density functions or responses that can be expressed as bounded probability density functions) provide the basis for characterizing the waveform. Traditionally, only the lower-order moments ( $k \leq 4)$ have been utilized in deriving topographical indices of these waveforms. Recent advances in waveform moment analysis, however, have made it possible to derive comprehensive and interpretable indices of complex nonnegative bounded waveforms by utilizing both lower-order and higher-order moments. Waveform moment analysis is reviewed briefly, and a flexible and efficient computer program is presented for conducting waveform moment analyses.
\end{abstract}

... in characterizing random functions, we have concentrated on the probability density function and what are called first and second moment functions.... These functions are particularly useful in that (a) they lend themselves to useful operations which permit interpretable results to be obtained; (b) they are achievable, although incomplete, descriptions which can be obtained experimentally; (c) they are compatible with linear systems analysis in a useful way.... It is reasonable to expect that as new information accumulates in this field, the analysis of complex waveforms will move in the direction of employing ... higher moment functions (Dern \& Walsh, 1963, p. 103).

Nonnegative bounded waveforms (hereafter referred to as NB waveforms) are defined as responses that have the following two general characteristics: (1) $f(x)$ and $x$ are bounded, and (2) $f(x) \geq 0$. This is a broad class of waveforms, including learning acquisition curves and integrated electromyographic responses in the time domain, amplitude distributions of the electroencephalogram and distributions of tests scores, and power spectra of various physical, behavioral, and bioelectric signals. Quantitative representations of NB waveforms have traditionally been

This research was supported by National Science Foundation Grant BNS-8414853 and by a grant of computing equipment from HewlettPackard to John T. Cacioppo. Correspondence may be addressed to John T. Cacioppo, Department of Psychology, Ohio State University, Columbus, $\mathrm{OH} 43210$, or to Beverly S. Marshall-Goodell or Louis G. Tassinary, Department of Psychology, University of lowa, Iowa City, IA 52242. insensitive to substantive features of the topography of those waveforms (see Cacioppo, Marshall-Goodell, \& Dorfman, 1983). Cacioppo and Dorfman (1987) recently developed a quantitative representation of NB waveforms predicated on the moments of the waveform in a given domain. Briefly, the size of the waveform is extracted, and the waveform is converted to a unit mass. An indicant based on the first moment of the waveform then provides the basis for a measure of central tendency of the response; indicants based on the higher-order even moments provide the basis for a multidimensional indicant of dispersion about a specified reference point (e.g., about the first moment or the midpoint); and indicants based on the higher-order odd moments provide the basis for a multidimensional indicant of asymmetry about the specified reference point. These indicants provide comprehensive and interpretable summaries of waveforms in the time, amplitude, frequency, and power domains.

Waveform moment analysis represents a model for the quantification of population parameters, not estimates of population parameters. Statistical dependence and independence are properties of sample estimates, not of population parameters. Therefore, no assumptions are made about the dependence or independence of the moments. However, because the moment-based indicants are likely to be correlated, we recommend the use of multivariate procedures for hypothesis testing. For details on these and other matters pertaining to estimation and hypothesis testing using moment-based indicants, see 
Cacioppo and Dorfman (1987). Importantly, despite the descriptive statistical nature of waveform moment analysis, the parameters derived in waveform moment analysis enable an investigator to draw specific conclusions about the size and form of the NB waveform, and to make inferences about possible underlying mechanisms responsible for the generation of the NB waveform. For instance, the larger the dispersion of an NB waveform in the amplitude domain, the greater the interrelatedness (i.e., positive correlation) among the underlying components responsible for the generation of the NB waveform (e.g., see Cacioppo \& Dorfman, 1987, pp. 432-433).

One potential limitation to waveform moment analysis is the time and effort involved in calculating multidimensional indicants based on higher-order moments. Our goal, therefore, is to outline an efficient, general purpose computer program for conducting waveform moment analyses. The Waveform Moment Analysis (WAMA) program is written in ASYST ${ }^{\mathrm{TM}}$, a commercially available structured programming language. WAMA can provide indicants of size, central tendency, asymmetry, and dispersion about one or more user-specified reference points in one or more domains (e.g., time, amplitude, frequency, power), and it is designed for any IBM PC, XT, AT or compatible microcomputer with at least $512 \mathrm{~K}$ and a math coprocessor. ASYST ${ }^{\mathrm{TM}}$ Modules 1 and 2 are also required. To enhance the portability of the program, summaries of the underlying structured programming logic are outlined below, and extensive documentation is provided within the program. ${ }^{1}$

Comprehension of the options, flexibility, and power of WAMA requires an understanding of waveform moment analysis. To facilitate our description of the WAMA program here, an abbreviated version of a tutorial provided by Cacioppo and Dorfman (1987), including extensions provided by Dorfman and Cacioppo (in press), follows.

\section{An Overview of Waveform Moment Analysis}

A distribution of bricks atop a beam, such as that illustrated in Figure 1, can be viewed as a physical example of an NB waveform. ${ }^{2}$ Assuming that gravity is constant, the force exerted at each position, $x_{i}$, along the beam equals the mass, $f\left(x_{i}\right)$, times a constant. Hence, the distribution in Figure 1 can be viewed as depicting points of force $\left[x_{i}, f\left(x_{i}\right)\right]$ where $x_{i}$ signifies some position along the beam, $f\left(x_{i}\right)$ is proportional to the force exerted at location $x_{i}$ along the beam, and $\alpha_{x}$ represents a fulcrum placed at some arbitrary position.

The moments of this distribution of bricks provide the basis for differentiating this from alternative distributions of bricks that might be placed atop the beam, and for identifying the manner in which this and an alternative distribution of bricks differ. ${ }^{3}$ To begin, notice that the tendency for any given stack of bricks to produce rotation of the beam is given by the expression $\left|x_{i}-\alpha_{x}\right| f\left(x_{i}\right)$, indicating that the tendency for any given stack of bricks to induce rotation about the fulcrum is proportional to: (1) the dis-



Figure 1. Stacks of bricks of mass $f\left(x_{i}\right)$ applied simultaneously at positions $x_{i}$ along a beam, which is supported by a fulcrum at $\alpha_{x}$ Adapted from J. T. Cacioppo and D. D. Dorfman (1987), "Waveform Moment Analysis in Psychophysiological Research," Psychological Bulletin, 102, 421-438.

tance from the fulcrum at which the stack of bricks is positioned (i.e., $\left.\left|x_{i}-\alpha_{x}\right|\right)$, and (2) the mass of the stack of bricks-i.e., $f\left(x_{i}\right)$. If $N$ stacks of bricks are simultaneously placed atop the beam at positions $x_{1}, x_{2}, \ldots x_{N}$, respectively, then the total tendency of this particular distribution of bricks to induce rotation is given by the equation:

$$
\sum_{i=1}^{N}\left(x_{i}-\alpha_{x}\right) f\left(x_{i}\right)=\mu_{1: x}^{\prime},
$$

the first moment about the arbitrary position, $\alpha_{x}$, of the fulcrum. In general, the $k$ th moment of this distribution of bricks about $\alpha_{x}$ is

$$
\mu_{k: x}^{\prime}=\sum_{i=1}^{N}\left(x_{i}-\alpha_{x}\right)^{k} f\left(x_{i}\right) .
$$

Conversion of the NB waveform to a unit mass is achieved by dividing the mass $f\left(x_{i}\right)$ of each stack of bricks by $\sum_{i=1}^{N} f\left(x_{i}\right)$. Equating NB waveforms with respect to their size enables a size-independent measure of the shape of the waveform. This allows comparisons among NB waveforms in terms of their size and their shapes. ${ }^{4}$

Furthermore, the sequence of moments about $\alpha_{x}$ obtained when $k$ is even $(k=2,4,6, \ldots)$ can be used to gauge the dispersion of the distribution of bricks about $\alpha_{x}$, and the sequence of moments about $\alpha_{x}$ obtained when $k$ is odd $(k=3,5,7, \ldots)$ can be used to gauge the asymmetry of the distribution of bricks about $\alpha_{x}$. For instance, given $f\left(x_{i}\right)>0$, for $x_{i}$ not equal to $\alpha_{x}$ and $k$ even, $\left(x_{i}-\alpha_{x}\right)^{k} f\left(x_{i}\right)>0$. Hence, for $k$ even, the moments are always positive except, of course, for the case in which $f\left(x_{i}\right)>0$ for $x_{i}=\alpha_{x}$ and $f\left(x_{i}\right)=0$ for all $x_{i}$ not equal to $\alpha_{x}$. Inspection of Equation 2 also reveals that the even moments are sensitive to the absolute deviations of the locations, $x_{i}$, of the stacks of bricks from $\alpha_{x}$. The greater the distance of the stacks from $\alpha_{x}$, the greater the impact on 
the moments. A useful consequence of these facts is that the even moments provide a mathematically rigorous manner of comparing the dispersion of various stacks of bricks when each stack is expressed in unit mass.

It is also easy to show by similar reasoning that the odd moments are zero if and only if the NB waveform is symmetric about $\alpha_{x}$, and that the odd moments about $\alpha_{x}$ provide a multidimensional gauge that provides information about the direction as well as the extent of asymmetry about $\alpha_{x}$ (see Cacioppo \& Dorfman, 1987).

Finally, the order of the moment is significant. It has been standard practice to consider only the lower-order moments (e.g., see Dern \& Walsh, 1963, pp. 102-104). Indices of asymmetry, for instance, have traditionally been limited to $k=3$ and $\alpha_{x}=\mu_{1: x}^{\prime}$ (i.e., the arithmetic mean), whereas indices of dispersion have traditionally been limited to variance (where $k=2$ and $\alpha_{x}=\mu_{1: x}^{\prime}$; see Dorfman $\&$ Cacioppo, in press). When dealing with simple (e.g., unimodal) waveforms, the classic shape descriptors of mean, variance, skewness, and kurtosis, which are based on the lower-order moments, often provide satisfactory characterizations of these waveforms; however, when dealing with more complex waveforms, these classic shape descriptors can provide nondiscriminating characterizations of clearly distinctive waveforms (see Cacioppo et al., 1983). With the ready availability of the high-speed digital computer and efficient computational procedures such as those provided by WAMA, one might profitably compute higher-order indicants of asymmetry and dispersion to derive more complete summaries of these features of NB waveforms. For instance, whether a waveform is characterized by positive or negative asymmetry depends on the reference point and the order of the moment selected (e.g., see Cacioppo \& Dorfman, 1987, pp. 434-437).

Several problems have limited the interpretability of the higher-order moments per se (see Dorfman \& Cacioppo, in press). Cacioppo and Dorfman (1987) have proposed that if, as in many instances in psychological research, one seeks to derive indicants with which to describe or compare the shape of NB waveforms, three transformations of Equation 2 are useful. The first, which we have already discussed, is to equate the waveforms with respect to the total mass by expressing each mass, $f\left(x_{i}\right)$, as a proportion of the total mass, $\sum_{i=1}^{N} f\left(x_{i}\right)$. This helps to uncouple the dimensions of response size and response shape.

Second, the $k$ th moment is taken to the $1 / k$ th power so that all of the moments are expressed in the original units of measurement such as centimeters, microvolts, microsiemans, milliseconds, etc. Note that taking the $1 / k$ th power of the $k$ th moment converts the even moments to true distance functions (Cacioppo \& Dorfman, 1987 ) and diminishes many of the early criticisms associated with the use of higher-order moments to characterize waveforms (Dorfman \& Cacioppo, in press). This transformation also has the effect of increasing the im- pact of $x_{i}$, relative to $f\left(x_{i}\right)$, on the moment-based indicants as $k$ increases.

Third, if the width of the domain of interest (e.g., the length of a beam or recording interval) varies, comparisons of the topography of NB waveforms in this domain are facilitated by introducing a scaling factor, $1 / \beta_{x}$. For instance, when dealing with beams of differing lengths, distances $x_{i}$ can be expressed as a proportion of the total distance prior to calculating the moment-based indicants. The total distance is the moment-based indicant about zero when $k=\infty$. Consider a hypothetical example in which symmetrical distributions of bricks peak midway along the beam in every instance, but the width of the distribution of bricks is twice as wide for some stacks of bricks as for others. By setting $\beta_{x}$ equal to the width of the distribution in each instance, and by extracting the width of the distribution as a separate parameter, the moment-based indicants of these NB waveforms provide a more interpretable depiction of their shape (e.g., the center of gravity is 0.5 along the width of the distribution in each stack of bricks). Thus, this scaling factor allows the investigator to distinguish between the topography and the length of an NB waveform in a particular domain (for more information, see Dorfman \& Cacioppo, in press).

In sum, the moment-based representation of the topography of a waveform such as the bricks atop the beam in Figure 1 can be stated as:

$$
\gamma_{k: x}^{\prime}=1 / \beta_{x}\left[\frac{\sum_{i=1}^{N}\left(x_{i}-\alpha_{x}\right)^{k} f\left(x_{i}\right)}{\sum_{i=1}^{N} f\left(x_{i}\right)}\right]^{1 / k}, k=1,2,3, \ldots
$$

The fact that asymmetry and dispersion are always stated in terms of a reference point may not be immediately obvious. Nor may it be obvious that, although we have treated $\alpha_{x}$ as an arbitrary reference point, the specification of the reference point is not arbitrary for the researcher since the reference point is fundamental to the interpretation of the indicants. When one thinks of an NB waveform as a physical entity such as a stack of bricks atop a beam, however, it is obvious that characterizations of this entity are dependent on the height and distance of each stack of bricks atop the beam relative to the fulcrum (reference point). Note, too, that: (1) the first moment will be zero when the fulcrum is placed at the abscissa of the center of gravity-that is, the first moment about zero; and (2) the higher-order moments with reference to a waveform-dependent reference point (e.g., the first moment about zero) are not equivalent to the higher-order moments with reference to a waveform-independent reference point (e.g., the midpoint of the abscissa).

Both waveform-dependent and waveform-independent reference points have important applications (see Cacioppo \& Dorfman, 1987). Traditional shape descriptors (e.g., variance, skewness, kurtosis) are calculated about a waveform-dependent reference point (e.g., the 
mean). A waveform-independent reference point can be useful, however, when comparing subtle differences in the shapes of two or more waveforms, for it yields a description of the asymmetry and dispersion with respect to a fixed point common to all subjects and conditions. Waveform-dependent reference points, on the other hand, yield unequal weights for each $f\left(x_{i}\right)$ except in the case in which the waveform-dependent reference points (e.g., centers of gravity) are themselves equal. In sum, the question asked determines which reference point is best, and Cacioppo and Dorfman's (1987) waveform moment analysis allows the investigator to specify this important parameter.

\section{Domains of Waveforms}

If the stacks of bricks are viewed as placed not at various positions across the beam, but rather at a given position on the beam at various intervals in time, then an NB waveform can be defined as the forces at that position across time. The amplitudes depicted in Figure 1, for instance, could represent a variety of measures in psychology, such as microvolts of physiological activity measured across several seconds, the amount of pressure exerted on a transducer (e.g., handgrip, microswitch) during a trial, or some feature (e.g., pitch, volume) of one's voice during an articulation. The reasoning underlying the indicants defined by Equation 3, therefore, can be extended in a straightforward fashion to the time domain, where $\alpha_{x}$ now represents a reference point in time and $\beta_{x}$ now represents a temporal scaling factor such as the length of the recording interval.

Both NB waveforms and arbitrary bounded waveforms in the time domain give rise to NB waveforms in the amplitude domain (i.e., amplitude distributions), which can be of interest in psychology (see, e.g., Cooper, Osselton, \& Shaw, 1980; Lippold, 1967). A number of important biological or behavioral measures in the time domain, for instance, have amplitudes that are bounded in all cases of practical interest but assume negative as well as nonnegative values (e.g., electroencephalogram). Because the amplitudes can assume negative values, such waveforms are not NB waveforms in the time domain, and waveform moment analysis in this domain are not appropriate. If, on the other hand, we consider the distribution of amplitudes, then waveform moment analysis are appropriate. The reasoning underlying the indicants defined by Equation 3 can be extended simply to the amplitude domain, where $\alpha_{x}$ now represents a reference point in the amplitude domain (e.g., mean amplitude), and $\beta_{x}$ now represents a scaling factor such as the standard deviation or the range of observed amplitudes when you have a very large sample size. Interpretation of these shape descriptors is a simple extension of our discussion above.

There are also a variety of waveforms that are usefully represented in the frequency domain, such as speech, body temperature, and the electroencephalogram. One begins with a collection of amplitudes observed sequentially in time-amplitudes that may take on negative as well as non- negative values. To obtain a frequency representation of that collection of observations, the power spectrum or spectral density function of that collection of observations is computed (e.g., see Bracewell, 1978; Porges \& Bohrer, in press). If there are a few frequencies where the power is almost totally concentrated, then the power spectrum can be characterized in terms of those few frequencies. If, on the other hand, the power spectrum is smeared over a broad spectral band in a rather smooth fashion, then the power spectrum cannot be fully characterized by a few frequencies. Note, however, that the power spectrum itself constitutes an NB waveform. Hence, to probe the topography of the power spectrum and to compare specific topographical features across power spectra, it may be useful to characterize the power spectrum in terms of the moment-based indicants described earlier using Equation 3 , where $x_{i}$ now represents a frequency, $f\left(x_{i}\right)$ represents a measure of power of the corresponding frequency bin, and $\alpha_{x}$ now represents a reference point in the frequency domain (see Cacioppo \& Dorfman, 1987; Dorfman \& Cacioppo, in press).

Finally, just as a waveform in the time domain gives rise to one and only one amplitude distribution that is an NB waveform, each power spectral density function can give rise to a power distribution that can be characterized using a waveform moment analysis. Hence, WAMA can provide detailed, quantitative information about the size and shape of a response in the time/distance, amplitude, frequency, or power domains from a variety of userspecified perspectives (reference points). Furthermore, the availability of a flexible, efficient, and economical computer program for calculating these indicants makes it feasible to conduct exploratory data analyses using various reference points.

\section{The Waveform Moment Analysis (WAMA) Program}

The general structure of the WAMA program is diagrammed in Figure 2, and a more detailed diagram representing the logical flow of the calculation of moments-based indicants is presented in Figure 3. WAMA operates on two input files: (1) a datafile, and (2) a parameter file. As noted above, waveform moment analysis provides the investigator with considerable flexibility in terms of specifying the domain(s) of an NB waveform to be analyzed, the reference point, the scaling factor, and the number of moment-based indicants to be extracted. The parameter file contains this user-specified programming information as well as information about the name, location, and format of the datafile (i.e., waveforms). A small datafile is illustrated in Appendix A, and a sample parameter file is displayed in Appendix B.

Datafiles. Each subject has associated with him/her $p$ datafiles, where $p$ represents the number of trials in the study. Each datafile, in turn, is composed of $q$ subfiles, where $q$ represents the number of distinct recording channels or input streams sampled during the trial. Each subfile consists of a digital array in the form $\left\{f\left(x_{1}\right), f\left(x_{2}\right) \ldots\right.$ $\left.f\left(x_{r}\right)\right\}$, ordered sequentially, where $r$ represents the num- 


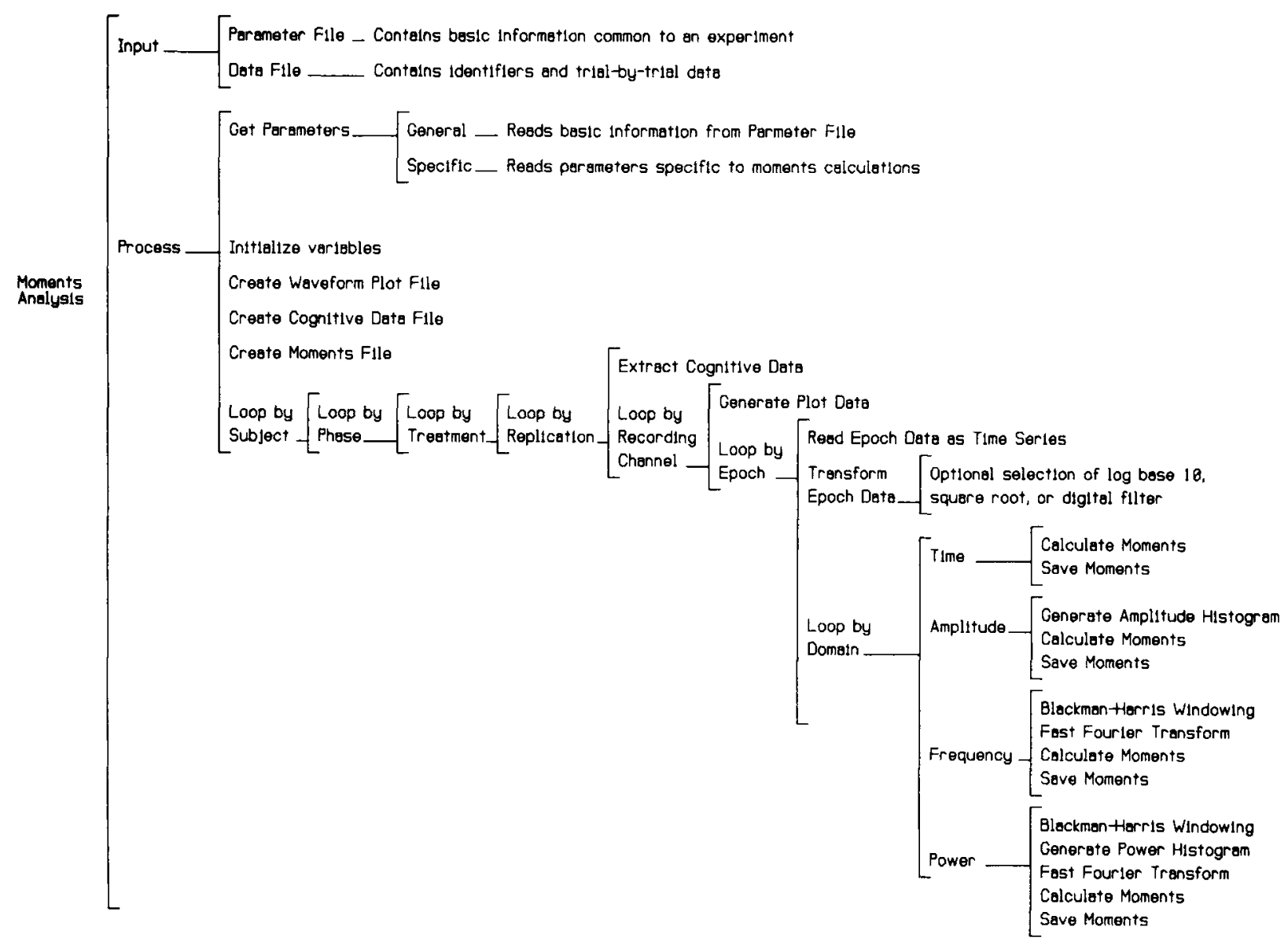

Figure 2. General structure of the WAMA computer program.

ber of observations obtained from a given recording channel and subject on a single recording trial (see Appendix A). Finally, each digital array can be partitioned into $s$ epochs, where $s$ represents the segment(s) of the digital array that constitutes the response to be subjected to waveform moment analysis. The values in each datafile are assumed to be stored as real, single precision numbers. All calculations depicted in Equation 3 are performed in double precision on the subjects, trials, subfiles, and epochs of the digital arrays specified by the investigator. As noted, this specification is done in the parameter file, to which we turn next.

Parameter file. The parameter file can be prepared using ASYST ${ }^{\mathrm{TM}}$ commands, but a special-purpose editor has been written and incorporated into WAMA to help the user create the parameter file needed in the subsequent portions of the program. Hence, WAMA is an interactive program in that it prompts the user to specify the information used in analyzing the datafile. Included among the information queried about the format of the datafile are the number of subjects, number of trials, number of recording channels, and the number of epochs per trial; the onset and offset of each recording epoch within a trial; and the sampling rate. To provide the user with the flexibility to analyze blocks of trials (or phases of an experi- ment) separately, the program also prompts the user to specify the number of phases in a study, and the number of trials of each treatment ("trial type") within a phase. The parameters governing analyses which are specified by the user include the domain(s) in which the moments are to be calculated (i.e., time, amplitude, frequency, power); the number of moments to be calculated; the reference point(s) (e.g., zero, mean, midpoint) about which the first moment, higher-order even moments, and higher-order odd moments are to be calculated; the scaling factor to be employed; and whether the output should be stored in an ASCII output file as well as in an ASYST $^{\mathrm{TM}}$ output datafile.

Options for data transformations (e.g., $\log$ base $_{10}$, square root) prior to input into WAMA are also provided (see Figure 3). If the user specifies in the parameter file that moment-based indicants are to be calculated in either the frequency or power domain, the specified portions of the datafiles in the time domain are automatically subjected to a minimum four-sample Blackman-Harris window (Harris, 1978; Nuttal, 1981; cf. Welch, 1967), and, subsequently, to the fast fourier transform subroutine in ASYST $^{\mathrm{TM}}$. The power spectral density function for each specified segment (i.e., epoch) then serves as the input datafile for WAMA. As noted in the preceding section, 


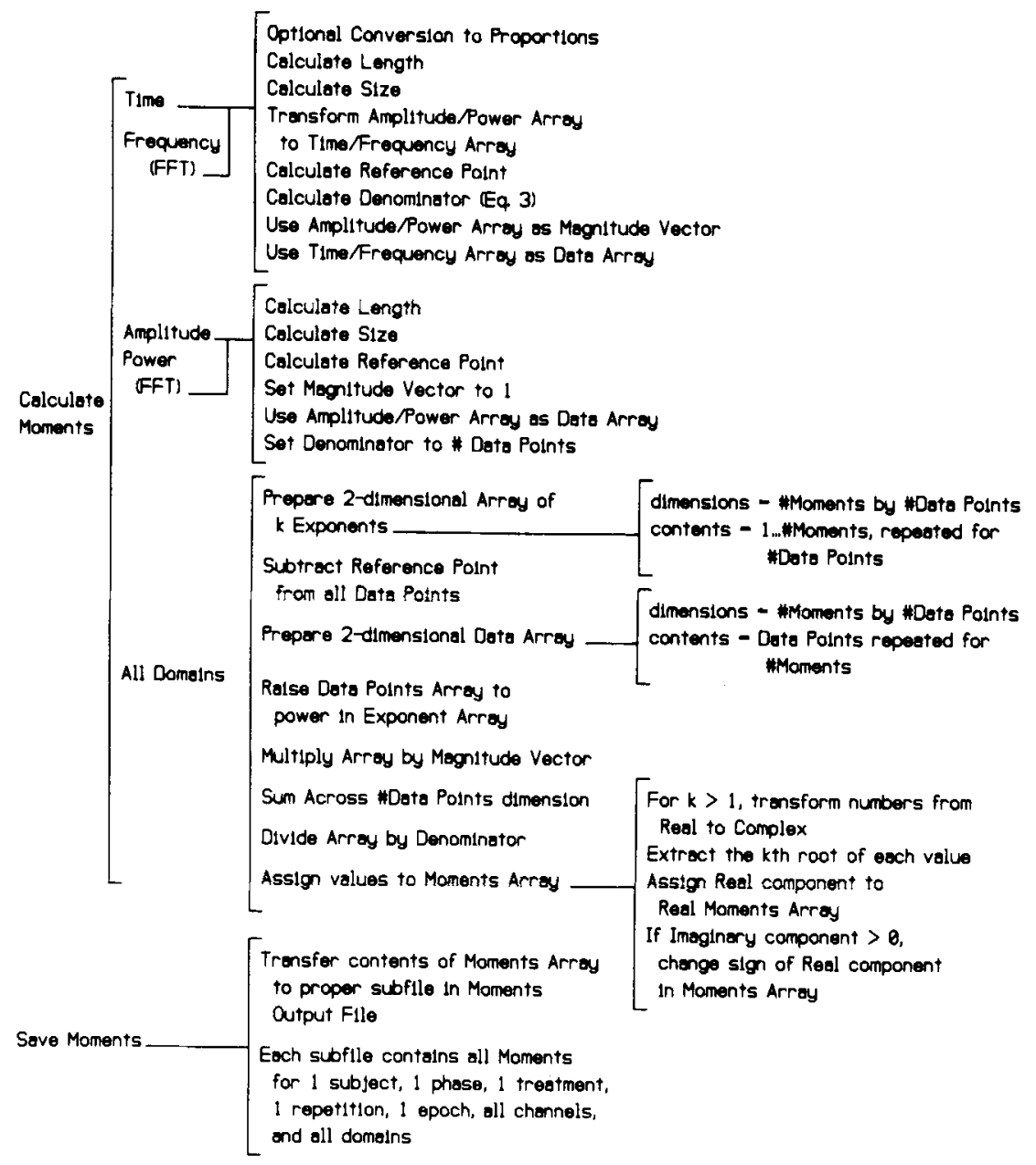

Figure 3. Specific outline and description of the waveform moment analysis. The algorithm used in calculating the moment-based indicants is of the form specified in Equation 3.

the power spectral density function constitutes an NB waveform, so its input to WAMA is automated and the interpretation of the resultant output is straightforward.

Output Files. WAMA is performed on a subject-bysubject basis, and the resulting output files are concatenated to create a dataset ready for inferential statistical analyses. Specifically, based on the data provided in the datafiles and the information provided in the parameter file, WAMA then calculates moment-based indicants for each specified epoch (e.g., waveform) of the digital arrays in the datafiles. If a portion of a digital array is submitted to WAMA but does not meet the definition of an NB waveform, an error code rather than an erroneous set of indicants is generated. Error codes are also stored for input datafiles not found by the program. As noted, the resulting indicants (and error codes) are then stored in a separate ASYST ${ }^{\mathrm{TM}}$ datafile for each subject and, if requested, in an output file in ASCII form (see Appendix C).

Although ASYST ${ }^{\mathrm{TM}}$ output files are created automatically by WAMA, it is the ASCII output files created for each subject that are usually of greater interest to the investigator. This is because the format of the ASCII out- put files are designed to be compatible with most statistical analysis (e.g., SPSS/PC+ ${ }^{\mathrm{TM}}$, ASYST ${ }^{\mathrm{TM}}, \mathrm{SAS}^{\mathrm{TM}}$, SYSTAT $^{\mathrm{TM}}$ ) and communications programs to simplify their concatenation (across subjects) and analysis. The general format for the ASCII output file for each subject is illustrated in Appendix C. Briefly, if $p$ is the total number of trials in the study, $q$ represents the number of distinct recording channels or input streams sampled during the trial, $s$ represents the number of epochs into which each array of data collected during a given trial on a given channel has been subdivided, and $d$ represents the number of domains in which waveform moment analyses are performed, then each ASCII output file is composed of $p^{*} q^{*} s^{*} d$ paragraphs of data. Each paragraph of data includes fields identifying the subject number, replication number, phase, trial type, trial number within trial type, epoch, recording channel, and domain of waveform moment analysis. Following this label within each paragraph of data are the WAMA indicants for central tendency $\left(\gamma_{1: x}^{\prime}\right)$, dispersion $\left(\gamma_{k: x}^{\prime}\right.$, for $k$ even $)$, and asymmetry $\left(\gamma_{k: x}^{\prime}\right.$, for $k$ odd), as defined in Equation 3, and the additional indices of the total mass $\sum_{i=1}^{N} f\left(x_{i}\right)$ and a scaling factor (e.g., $\left.\beta_{x}\right)$. 


\section{Statistical Inferences}

WAMA is performed on individual responses on a subject-by-subject basis, and the resulting output files are concatenated to create a dataset ready for inferential statistical analyses where moment-based indicants of individual responses are the observations. That is, WAMA represents an individual subject's reponse (or each of a subject's responses) by a vector of moment-based indicants. The final goal of an investigation is not usually data reduction or data representation, but statistical inference. For purposes of statistical inference, replications within subjects provide the basis for estimation of within-subject variability and replications between subjects provide the basis for estimation of between-subjects variability (Cacioppo \& Dorfman, 1987). Under the assumption of the robustness of Gaussian statistics, statistical tests derived from classical univariate and multivariate normal distribution theory should be appropriate. On the other hand, there are methods for interval estimation and hypothesis testing that are distribution-free-that is, they are not based upon classical Gaussian distribution theory-and are yet powerful. One such technique, the jackknife technique, can be applied to the moment-based indicants if one wishes to avoid classical Gaussian distribution theory (see Arvesen \& Salsburg, 1975; Efron, 1982; Rey, 1978; Tukey, 1969). Two very readable introductions to the jackknife technique-written for psychologists-can be found in Mosteller and Tukey's (1968) chapter and in Tukey's (1969) article. Recent applications of the jackknife method to problems of statistical inference can be found in Faraone and Dorfman (1987).

To summarize, waveform moment analyses of NB waveforms in the time, amplitude, frequency, or power domains provide a systematic means of representing both simple and complex responses. This approach not only offers the advantage of reducing the data constituting the "response"' but also allows rigorous intra-individual and interindividual comparisons. Two NB waveforms are identical, for instance, if and only if all their moments and their total mass are equal. The WAMA program simplifies the calculation of these moment-based indicants and provides investigators with flexibility in specifying the domains of analysis, reference points, number of moments, and scaling factors to be used.

\section{REFERENCES}

ARVeSEn, J. N., \& SALSBURG, D. S. (1975). Approximate tests and confidence intervals using the jackknife. In R. M. Elashoff (Ed.), Perspectives in biometrics (Vol. 1, pp. 123-147). New York: Academic Press.

Bracewell, R. N. (1978). The Fourier transform and its applications (2nd ed.). New York: McGraw-Hill.

CACIOPPO, J. T., \& DorfMAN, D. D. (1987). Waveform moment anal- ysis in psychophysiological research. Psychological Bulletin, 102, $421-438$.

Cacioppo, J. T., Marshall-Goodell, B. S., Dorfman, D. D. (1983). Skeletomuscular patterning: Topographical analysis of the integrated electromyogram. Psychophysiology, 20, 269-283.

Cooper, R., Osselton, J. W., Shaw, J. C. (1980). EEG technology (3rd ed.). London: Butterworths.

DERN, H., WALSH, J. B. (1963). Analysis of complex waveforms. In W. L. Nastuk (Ed.), Physical techniques in biological research: Vol. VI. Electrophysiological methods, Pt. B (pp. 99-219). New York: Academic Press.

DorfmaN, D. D., \& CACIOPPO, J. T. (in press). Waveform moment analysis: Topographical analysis of nonrhythmic waveforms. In J. T. Cacioppo \& L. G. Tassinary (Eds.), Principles of psychophysiology: Physical, social, and inferential elements. New York: Cambridge University Press.

EFron, B. (1982). The jackknife, the bootstrap, and other resampling plans. Philadelphia: Society for Industrial and Applied Mathematics.

Faraone, S. V., \& Dorfman, D. D. (1987). Lag sequential analysis: Robust statistical methods. Psychological Bulletin, 101, 312-323.

Harrus, F. J. (1978). On the use of windows for harmonic analysis with the discrete Fourier transform. Proceedings of the IEEE, 66, 51-83. LiPPoLD, O. C. J. (1967). Electromyography. In P. H. Venables \& I. Martin (Eds.), Manual of psychophysiological methods (pp. 245298). New York: Wiley.

Mosteller, F., TUkEY, J. W. (1968). Data analysis including statistics. In G. Lindzey \& E. Aronson (Eds.), Handbook of social psychology (Vol. 2, pp. 80-203). Reading, MA: Addison-Wesley.

Nutral, A. H. (1981). Some windows with very good sidelobe behavior. IEEE Transactions on Acoustics, Speech, and Signal Processing, ASP-29, 85-91.

Porges, S. W., Bohrer, R. E. (in press). Analyses of periodic processes in psychophysiological research. In J. T. Cacioppo \& L. G. Tassinary (Eds.), Principles of psychophysiology: Physical, social, and inferential elements. New York: Cambridge University Press. REY, W. J. J. (1978). Robust statistical methods. New York: SpringerVerlag.

TUKEY, J. W. (1969). Analyzing data: Sanctification or detective work? American Psychologist, 24, 83-91.

WeLch, P. D. (1967). The use of fast Fourier transform for the estimation of power spectra: A method based on time-averaging over short modified periodograms. IEEE Transactions on Audio and Electroacoustics, AU-15, 70-73.

\section{NOTES}

1. The program listing, which extends approximately 50 pages, is available from the second author upon request. A simple but much more limited program written in BASIC to illustrate waveform moment analysis is also available from Louis $\mathbf{G}$. Tassinary upon request.

2. The distribution of data depicted in Figure 1 could, of course, represent a variety of types of data (e.g., physiological signals, test scores, behavioral counts, etc.). The present concrete example is used only for didactic purposes.

3. A mathematical proof for these statements is provided in the appendices to Dorfman and Cacioppo (in press).

4. It should be emphasized that the classic shape descriptors of mean, variance, skewness, and kurtosis are based on the moments (see Cacioppo \& Dorfman, 1987). For instance, the abscissa of the center of gravity is simply an arithmetic mean. It can also be shown that if the moments of two equal-mass NB waveforms are similar, the waveforms are similar; if the moments are dissimilar, the waveforms are dissimilar (see Dorfman \& Cacioppo, in press). 


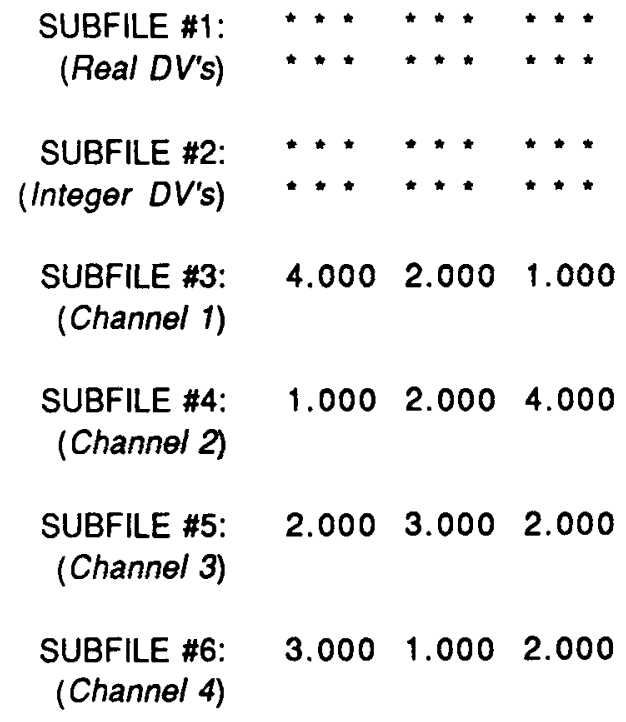

Three letter experiment code:

Name of Experimenter:

Number of Replications:

Number of Phases:

Number of Epochs:

Number of analog data channels:

Number of digital dependent variables

Real (e.g., RT):

Integer (e.g., rating scales):

Plotfile parameter:

Plotfile size:

Data Transformations:

Number of moments $(\gamma)$ :

Domains:

Analog Input 1: LABEL

Board Name:

ASYST board number:

Channel Number:

Sampling Rate $(\mathrm{Hz})$ :

Fullscale value (real units):

Output file subfile number:

Analog Input 3: LABEL

Board Name:

ASYST board number:

Channel Number:

Sampling Rate $(\mathrm{Hz})$ :

Fullscale value (real units):

Output file subfile number:
TES

Stacy Siegel

1

1

1

4

0

0

Unweighted Mean a

3

None

7

Time

Amplitude
DASH16A

Board Name:

ASYST board number:

Channel Number:

Sampling Rate $(\mathrm{Hz})$ :

Fullscale value (real units):

Output file subfile number:

Analog Input 4: LABEL

Board Name:

ASYST board number:

Channel Number:

Sampling Rate $(\mathrm{Hz})$ :

Fullscale value (real units):

Output file subfile number:
DASH16A 
REPLICATION 1: LABEL

PHASE 1: $L A B E L$

TRIAL TYPE 1: LABEL

Number of Trials:

Trial Duration:

1

3.000

EPOCH 1: LABEL

$\begin{array}{lr}\text { Start time (sec): } & .000 \\ \text { Stop time (sec): } & 3.000\end{array}$

CHANNELS 1-4: LABEL

DOMAINS:

Time:

Reference Points $(\alpha)$ :

Central Tendency: Origin

Dispersion:

Mean

Asymmetry Moan

Scale Factor $(\beta)$ : Original Units $(s e c)^{b}$

Amplitude:

Reference Points:

Central Tendency: Origin

Dispersion: Origin

Asymmetry: Origin

Scale Factor $(\beta)$ : $\quad$ Original Units $(\mu V)^{b}$

\section{APPENDIX C}

ASCII Output File ("D:SAMPLE.MOM")

Input file name:

Output file name:

Parameter file name:

Plotfile parameter:

Plotfile size:

Subject \#:

Replications:

Phases:

Trial Types:

Trial Number:

Epochs:

Channels:

Domains:

Type of Analysis:

Data Transformation:

Number of moments $(\gamma)$ :
C:Sample.dat

D:Sample.mom

C:Sample.par

Unweighted Meana

3

1

LABEL

LABEL

LABEL

LABEL

LABEL

LABEL

LABEL

LABEL

LABEL

Time

Amplitude

WAMA

None

7 
Trial Type 1: LABEL

Number of Trials:

Trial Duration:

Epoch 1: LABEL

Start time (sec): $\quad \quad .000$

Stop time (sec): $\quad 3.000$

Domains:

Time Domain:

Reference Points $(\alpha)$ :

Central Tendency:

Dispersion:

Asymmetry:

Scale factor $(\beta)$ :

Amplitude Domain:

Reference Points $(\alpha)$ :

Central Tendency:

Dispersion:

Asymmetry:

Scale factor $(\beta)$ :

3.000

(3.000

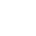

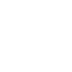


APPENDIX C (Continued)

$\begin{array}{crrrrrrr}\text { SUBNUM: } & 1.000 & 1.000 & 1.000 & 1.000 & 1.000 & 1.000 & 4.000 \\ \text { DOMAIN: } & 1.000 & 2.000 & .926 & .962 & .975 & 2.359 & 2.556 \\ \text { ASYM-3: } & 2.666 & 7.000 & 3.000 & & & & \\ & & & & & & & \\ \text { SUBNUM: } & 1.000 & 1.000 & 1.000 & 1.000 & 1.000 & 1.000 & 4.000 \\ \text { DOMAIN: } & 2.000 & 2.333 & 2.516 & 2.715 & 2.804 & 2.637 & 2.768 \\ \text { ASYM-3: } & 2.831 & 2.000 & 3.000 & & & & \end{array}$

a The Plotfile is an additional output file which is specifically designed to facilitate the visual inspection and ensemble averaging of the original waveforms. The WAMA program allows the investigator to specify the number of time bins $(\leq n)$ and replace the original values in each bin with either an unweighted mean or a weighted mean when plotting the response.

b The designation "original units" means $\beta=1$; that is, no scaling. 